\title{
EXISTENCE OF MILD SOLUTIONS OF SECOND-ORDER NEUTRAL FUNCTIONAL DIFFERENTIAL INCLUSIONS WITH NONLOCAL CONDITIONS IN BANACH SPACES
}

\author{
S. MARSHAL ANTHONI, J.-H. KIM, and J. P. DAUER
}

Received 15 October 2003

\begin{abstract}
We study the existence of mild solutions of the nonlinear second-order neutral functional differential and integrodifferential inclusions with nonlocal conditions in Banach spaces. The results are obtained by using the theory of strongly continuous cosine families of bounded linear operators and a fixed point theorem for condensing maps due to Martelli.
\end{abstract}

2000 Mathematics Subject Classification: 26A33.

1. Introduction. In the two recent decades, existence problem for differential inclusions has attracted much attention. Differential inclusions represent an important generalization of differential equations. The solution of a differential inclusion is a reachable set instead of a single trajectory. Ordinary and partial differential equations as modeling tools are too primitive to describe the behavior of many real dynamical systems. The differential inclusion is the most appropriate tool in dynamic uncertainty treatment. So it becomes important to study the qualitative behaviors of differential and integrodifferential inclusions. In recent years, the existence results for differential and integrodifferential inclusions have been extensively studied by several authors.

In this work, we prove the existence of mild solutions for second-order neutral functional differential and integrodifferential inclusions in Banach spaces. In Section 3, we consider the second-order neutral functional differential inclusion

$$
\begin{array}{cc}
\frac{d}{d t}\left[x^{\prime}(t)-g\left(t, x_{t}\right)\right] \in A x(t)+F\left(t, x_{t}, x^{\prime}(t)\right), & \text { a.e. } t \in J=[0, T], \\
x(t)+h_{t}(x)=\phi(t), \quad t \in J_{0}=[-r, 0], & x^{\prime}(0)=y_{0},
\end{array}
$$

where the state $x(\cdot)$ takes values in a real Banach space $X$ with the norm $|\cdot|, \phi \in$ $C\left(J_{0}, X\right), y_{0} \in X, A$ is the infinitesimal generator of a strongly continuous cosine family $\{C(t): t \in \mathbb{R}\}, g: J \times C\left(J_{0}, X\right) \rightarrow X, h_{t}: C\left(J_{0}, X\right) \rightarrow X, t \in J_{0}$, are given functions, and $F: J \times C\left(J_{0}, X\right) \times X \rightarrow 2^{X}$ is a bounded, closed, convex multivalued map.

For a continuous function $x$ defined on the interval $J_{1}=[-r, T]$ and $t \in J$, we denote by $x_{t}$ the element of $C\left(J_{0}, X\right)$ defined by

$$
x_{t}(s)=x(t+s), \quad s \in J_{0} .
$$

Here $x_{t}(\cdot)$ represents the history of the state from time $t-r$ up to the present time $t$. 
We refer the reader to $[9,10,14]$ and the references cited therein for motivation regarding the nonlocal initial conditions. As indicated in the above papers, the nonlocal condition $x(0)+g(x)=x_{0}$ can be applied in physics with better effect than the classical Cauchy problem $x(0)=x_{0}$. For example, $h_{t}(x)$ may be given by

$$
h_{t}(x)=\sum_{i=1}^{p} c_{i} x\left(t_{i}+t\right), \quad t \in J_{0},
$$

where $c_{i}, i=1, \ldots, p$, are given constants and $0<t_{1}<t_{2}<\cdots<t_{p} \leq T$. At time $t=0$, we have

$$
h_{0}(x)=\sum_{i=1}^{p} c_{i} x\left(t_{i}\right)
$$

In this case, it allows the measurements at $t_{i}, i=1, \ldots, p$, rather than just at $t=0$.

In Section 4, we study the existence of mild solutions for second-order neutral functional integrodifferential inclusion

$$
\begin{gathered}
\frac{d}{d t}\left[x^{\prime}(t)-g\left(t, x_{t}\right)\right] \in A x(t)+\int_{0}^{t} K(t, s) F\left(s, x_{s}, x^{\prime}(s)\right) d s, \quad \text { a.e. } t \in J=[0, T], \\
x(t)+h_{t}(x)=\phi(t), \quad t \in J_{0}=[-r, 0], \quad x^{\prime}(0)=y_{0},
\end{gathered}
$$

where $A, F, g, h_{t}$, and $\phi$ are as in the problem (1.1) and $K: D \rightarrow \mathbb{R}, D=\{(t, s) \in J \times J$ : $t \geq s\}$.

In many cases, it is advantageous to treat the second-order abstract differential equations directly rather than to convert them into first-order systems. A useful tool for the study of abstract second-order equations is the theory of strongly continuous cosine families. Here we use some of the basic ideas from cosine family theory. We refer to $[30,31]$ for a detailed discussion of cosine family theory. Second-order equations which appear in a variety of physical problems can be found in [4, 17].

For existence results on nonlocal initial value problem (IVP), we refer to the papers of Byszewski [11], Balachandran and Chandrasekaran [2, 3], Dauer and Balachandran [12], Lin and Liu [23], and Ntouyas and Tsamatos [25, 26]. Existence results for differential inclusions on compact intervals are given in the papers of Avgerinos and Papageorgiou [1] and Papageorgiou [27, 28]. In [6], Benchohra and Ntouyas studied the existence results for second-order differential inclusions on noncompact intervals.

This paper is motivated by the recent papers of Benchohra and Ntouyas [7] and Hernández and Henríquez [19, 20].

2. Preliminaries. In this section, we collect some basic facts from multivalued analysis which will be used in this paper.

Let $C(J, X)$ be the Banach space of continuous functions from $J$ into $X$ with the norm

$$
\|x\|_{\infty}:=\sup \{|x(t)|: t \in J\} .
$$


Let $B(X)$ denote the Banach space of bounded linear operators from $X$ into $X$. A measurable function $x: J \rightarrow X$ is Bochner integrable if and only if $|x|$ is Lebesgue integrable. (For properties of the Bochner integral see Yosida [32].)

Let $L^{1}(J, X)$ denotes the Banach space of continuous functions $x: J \rightarrow X$ which are Bochner integrable, with the norm

$$
\|x\|_{L^{1}}=\int_{0}^{T}|x(t)| d t \quad \forall x \in L^{1}(J, X)
$$

Let $(X,|\cdot|)$ be a Banach space. A multivalued map $G: X \rightarrow 2^{X}$ is convex (closed) valued if $G(x)$ is convex (closed) for all $x \in X$.

$G$ is bounded on bounded sets if $G(D)=\bigcup_{x \in D} G(x)$ is bounded in $X$, for any bounded set $D$ of $X$, that is,

$$
\sup _{x \in D}\{\sup \{|y|: y \in G(x)\}\}<\infty .
$$

A map $G$ is called upper semicontinuous on $X$ if, for each $x_{0} \in X$, the set $G\left(x_{0}\right)$ is a nonempty closed subset of $X$ and if for each open set $V$ of $X$ containing $G\left(x_{0}\right)$, there exists an open neighborhood $U$ of $x_{0}$ such that $G(U) \subseteq V$.

A map $G$ is said to be completely continuous if $G(D)$ is relatively compact for every bounded subset $D \subseteq X$.

If the multivalued map $G$ is completely continuous with nonempty compact values, then $G$ is upper semicontinuous if and only if $G$ has a closed graph, that is, for $x_{n} \rightarrow x_{*}$, $y_{n} \rightarrow y_{*}$, with $y_{n} \in G x_{n}$, we have $y_{*} \in G x_{*}$.

The map $G$ has a fixed point if there is $x \in X$ such that $x \in G x$.

In the following, $\mathrm{BCC}(X)$ denotes the set of all nonempty bounded closed and convex subsets of $X$.

A multivalued map $G: J \rightarrow \operatorname{BCC}(X)$ is said to be measurable if for each $x \in X$, the function $Y: J \rightarrow \mathbb{R}$ defined by

$$
Y(t)=d(x, G(t))=\inf \{|x-y|, y \in G(t)\}
$$

belongs to $L^{1}(J, \mathbb{R})$.

An upper semicontinuous map $G: X \rightarrow 2^{X}$ is said to be condensing if, for any bounded subset $D \subseteq X$, with $\alpha(D) \neq 0$, we have

$$
\alpha(G(D))<\alpha(D)
$$

where $\alpha$ denotes the Kuratowski measure of noncompactness. For properties of the Kuratowski measure, we refer to Banaś and Goebel [5]. We remark that a completely continuous multivalued map is the easiest example of a condensing map. For more details on multivalued maps, see the books of Deimling [13] and $\mathrm{Hu}$ and Papageorgiou [21]. 
Next, we give some basic results regarding the cosine families of bounded linear operators. We say that the family $\{C(t): t \in \mathbb{R}\}$ of operators in $B(E)$ is a strongly continuous cosine family if

(i) $C(0)=I$ is the identity operator in $E$;

(ii) $C(t+s)+C(t-s)=2 C(t) C(s)$ for all $s, t \in \mathbb{R}$;

(iii) the map $t \rightarrow C(t) x$ is strongly continuous for each $x \in X$.

The strongly continuous sine family $\{S(t): t \in \mathbb{R}\}$, associated to the given strongly continuous cosine family $\{C(t): t \in \mathbb{R}\}$, is defined by

$$
S(t) x=\int_{0}^{t} C(s) x d s, \quad x \in X, t \in \mathbb{R} .
$$

Assume the following condition on $A$.

(H1) $A$ is the infinitesimal generator of a strongly continuous cosine family $C(t)$, $t \in \mathbb{R}$, of bounded linear operators from $X$ into itself and the adjoint operator $A^{*}$ is densely defined, that is, $\overline{D\left(A^{*}\right)}=X^{*}$ (see [8]).

The infinitesimal generator of a strongly continuous cosine family $C(t), t \in \mathbb{R}$, is the operator $A: X \rightarrow X$ defined by

$$
A x=\left.\frac{d^{2}}{d t^{2}} C(t) x\right|_{t=0}, \quad x \in D(A),
$$

where $D(A)=\{x \in X: C(t) x$ is twice continuously differentiable in $t\}$.

Define $E=\{x \in X: C(t) x$ is once continuously differentiable in $t\}$.

To establish our main theorem, we need the following lemmas.

LEMMA 2.1 (see [30]). Let (H1) hold. Then

(i) there exist constants $M \geq 1$ and $\omega \geq 0$ such that $|C(t)| \leq M e^{\omega|t|}$ and $\mid S(t)-$ $S\left(t^{*}\right)|\leq M| \int_{t}^{t^{*}} e^{\omega|s|} d s \mid$ for $t, t^{*} \in \mathbb{R}$;

(ii) $S(t) X \subset E$ and $S(t) E \subset D(A)$ for $t \in \mathbb{R}$;

(iii) $(d / d t) C(t) x=A S(t) x$ for $x \in E$ and $t \in \mathbb{R}$;

(iv) $\left(d^{2} / d t^{2}\right) C(t) x=A C(t) x$ for $x \in D(A)$ and $t \in \mathbb{R}$.

LEMмA 2.2 (see [30]). Let (H1) hold, let $v: \mathbb{R} \rightarrow X$ such that $v$ is continuously differentiable, and let $q(t)=\int_{0}^{t} S(t-s) v(s) d s$. Then

(i) $q$ is twice continuously differentiable and for $t \in \mathbb{R}, q(t) \in D(A)$,

(ii) $q^{\prime}(t)=\int_{0}^{t} C(t-s) v(s) d s$ and $q^{\prime \prime}(t)=A q(t)+v(t)$.

For more details on strongly continuous cosine and sine families, we refer the reader to the books of Goldstein [18] and to the papers of Fattorini [15, 16] and of Travis and Webb [30, 31].

To prove the existence result, we rely on the following fixed point theorem due to Martelli.

LEMMA 2.3 (see [24]). Let $X$ be a Banach space and $N: X \rightarrow \mathrm{BCC}(X)$ a condensing map. If the set

$$
\Omega:=\{x \in X: \lambda x \in N x \text { for some } \lambda>1\}
$$

is bounded, then $N$ has a fixed point. 
3. Second-order neutral differential inclusions. To obtain the existence result for the problem (1.1), we assume the following.

(H2) $C(t), t>0$, is compact.

(H3) $F: J \times C\left(J_{0}, X\right) \times X \rightarrow \mathrm{BCC}(X) ;(t, u, v) \rightarrow F(t, u, v)$ is measurable with respect to the first argument and upper semicontinuous with respect to the last two arguments. Also for each fixed $x \in C([-r, T], X) \cap C^{1}(J, X)$, the set

$$
S_{F, x}=\left\{f \in L^{1}(J, X): f(t) \in F\left(t, x_{t}, x^{\prime}(t)\right) \text { for a.e. } t \in J\right\}
$$

is nonempty.

(H4) The function $g: J \times C\left(J_{0}, X\right) \rightarrow X$ is completely continuous and for any bounded set $K$ in $C\left(J_{1}, X\right)$, the set $\left\{t \rightarrow g\left(t, x_{t}\right): x \in K\right\}$ is equicontinuous in $C(J, X)$ and there exist constants $c_{1}$ and $c_{2}$ such that

$$
|g(t, u)| \leq c_{1}\|u\|+c_{2}, \quad t \in J, u \in C\left(J_{0}, X\right) .
$$

(H5) $h_{t}: C\left(J_{0}, X\right) \rightarrow X, t \in J_{0}$, and there exists a constant $G>0$ such that

$$
\left\|h_{t}(x)\right\| \leq G \quad \text { for } x \in C\left(J_{0}, X\right), t \in J_{0},
$$

and the set

$$
\left\{x(0): x \in C\left(J_{0}, X\right),\|x\| \leq k, x(0)=\phi(0)-h_{0}(x)\right\}
$$

is precompact in $X$.

(H6) $\|F(t, u, v)\|:=\sup \{|w|: w \in F(t, u, v)\} \leq p(t) \psi(\|u\|+|v|)$ for almost all $t \in J$, $u \in C\left(J_{0}, X\right)$, and $v \in X$, where $p \in L^{1}\left(J, \mathbb{R}_{+}\right)$and $\psi: \mathbb{R}_{+} \rightarrow(0, \infty)$ is continuous and increasing with

$$
\int_{0}^{T} m(s) d s<\int_{\mathcal{c}}^{\infty} \frac{d s}{s+\psi(s)}<\infty
$$

where

$$
\begin{gathered}
M=\sup \{|C(t)|: t \in J\}, \quad M^{*}=\sup \{|A S(t)|: t \in J\}, \\
m(t)=\max \left\{M c_{1}+M c_{1}^{2}+M^{*} c_{1},\left(M+M T+M c_{1} T\right) p(t)\right\}, \\
c=\left(M+M c_{1}+M^{*}\right)(\|\phi\|+G)+\left(M+M T+M T c_{1}\right)\left[\left|y_{0}\right|+c_{1}(\|\phi\|+G)+c_{2}\right] \\
+\left(1+c_{1}\right) M T c_{2}+M^{*} T c_{2}+c_{2} .
\end{gathered}
$$

REMARK 3.1. (i) If $\operatorname{dim} X<\infty$, then for each $v \in C\left(J_{0}, X\right), S_{F, u} \neq \phi$ (see Lasota and Opial [22]).

(ii) $S_{F, u}$ is nonempty if and only if the function $Y: J \rightarrow \mathbb{R}$ defined by

$$
Y(t):=\inf \{|v|: v \in F(t, u)\}
$$

belongs to $L^{1}(J, \mathbb{R})$ (see Papageorgiou [27]). 
In order to define a mild solution for the problem (1.1), we consider the integral equation

$$
\begin{aligned}
x(t)= & C(t)\left[\phi(0)-h_{0}(x)\right]+S(t)\left[y_{0}-g\left(0, x_{0}\right)\right] \\
& +\int_{0}^{t} C(t-s) g\left(s, x_{s}\right) d s+\int_{0}^{t} S(t-s) f(s) d s, \quad t \in J,
\end{aligned}
$$

where

$$
f \in S_{F, x}=\left\{f \in L^{1}(J, X): f(t) \in F\left(t, x_{t}, x^{\prime}(t)\right) \text { for a.e. } t \in J\right\} .
$$

DEFINITION 3.2. A function $x:[-r, T] \rightarrow X, T>0$, is called a mild solution of the problem (1.1) if $x(t)+h_{t}(x)=\phi(t), t \in[-r, 0]$, and there exists a $v \in L^{1}(J, X)$ such that $v(t) \in F\left(t, x_{t}, x^{\prime}(t)\right)$ a.e. on $J$, and the integral equation (3.8) is satisfied.

We need the following lemma to prove our main theorem.

LEMMA 3.3 (see [22]). Let I be a compact real interval and let $X$ be a Banach space. Let $F$ be a multivalued map satisfying (H3) and let $\Gamma$ be a linear continuous mapping from $L^{1}(I, X)$ to $C(I, X)$. Then, the operator

$$
\Gamma \circ S_{F}: C(I, X) \longrightarrow \mathrm{BCC}(C(I, X)), \quad x \longrightarrow\left(\Gamma \circ S_{F}\right)(x)=\Gamma\left(S_{F, x}\right),
$$

is a closed graph operator in $C(I, X) \times C(I, X)$.

Now, we are able to state and prove our main theorem.

TheOREM 3.4. Assume that Hypotheses (H1)-(H6) are satisfied. Then system (1.1) has at least one mild solution on $J_{1}$.

Proof. Consider the space $Z=C([-r, T], X) \cap C^{1}(J, X)$ with the norm

$$
\|x\|^{*}=\max \left\{\|x\|_{J_{1}},\left\|x^{\prime}\right\|_{J}\right\}
$$

where

$$
\|x\|_{J_{1}}=\sup \{|x(t)|:-r \leq t \leq T\}, \quad\left\|x^{\prime}\right\|_{J}=\sup \left\{\left|x^{\prime}(t)\right|: 0 \leq t \leq T\right\} .
$$

Now, we transform the problem into a fixed point problem. Consider the multivalued map $N: Z \rightarrow 2^{Z}$ defined by $N x$ the set of functions $u \in Z$ such that

$$
u(t)= \begin{cases}\phi(t)-h_{t}(x) & \text { if } t \in J_{0}, \\ C(t)\left[\phi(0)-h_{0}(x)\right]+S(t)\left[y_{0}-g\left(0, x_{0}\right)\right] & \\ +\int_{0}^{t} C(t-s) g\left(s, x_{s}\right) d s+\int_{0}^{t} S(t-s) f(s) d s & \text { if } t \in J,\end{cases}
$$

where (3.9) holds. We remark that the fixed points of $N$ are mild solutions to (1.1). 
We will show that $N$ is completely continuous with bounded closed convex values and it is upper semicontinuous. The proof will be given in several steps.

STEP 1. $N x$ is convex for each $x \in Z$.

Indeed, if $u_{1}, u_{2}$ belong to $N x$, then there exist $f_{1}, f_{2} \in S_{F, x}$ such that, for each $t \in J$, we have

$$
\begin{aligned}
u_{1}(t)= & C(t)\left[\phi(0)-h_{0}(x)\right]+S(t)\left[y_{0}-g\left(0, x_{0}\right)\right] \\
& +\int_{0}^{t} C(t-s) g\left(s, x_{s}\right) d s+\int_{0}^{t} S(t-s) f_{1}(s) d s, \\
u_{2}(t)= & C(t)\left[\phi(0)-h_{0}(x)\right]+S(t)\left[y_{0}-g\left(0, x_{0}\right)\right] \\
& +\int_{0}^{t} C(t-s) g\left(s, x_{s}\right) d s+\int_{0}^{t} S(t-s) f_{2}(s) d s .
\end{aligned}
$$

Let $0 \leq \alpha \leq 1$. Then, for each $t \in J$, we have

$$
\begin{aligned}
\left(\alpha u_{1}+(1-\alpha) u_{2}\right)(t)= & C(t)\left[\phi(0)-h_{0}(x)\right]+S(t)\left[y_{0}-g\left(0, x_{0}\right)\right]+\int_{0}^{t} C(t-s) g\left(s, x_{s}\right) d s \\
& +\int_{0}^{t} S(t-s)\left[\alpha f_{1}(s)+(1-\alpha) f_{2}(s)\right] d s .
\end{aligned}
$$

Since $S_{F, x}$ is convex (because $F$ has convex values), then

$$
\alpha u_{1}+(1-\alpha) u_{2} \in N x
$$

STEP 2. $N$ maps bounded sets into bounded sets in $Z$.

Indeed, it is enough to show that there exists a positive constant $\ell$ such that, for each $u \in N x, x \in B_{q}=\left\{x \in Z:\|x\|^{*} \leq q\right\}$, one has $\|u\|^{*} \leq \ell$. If $u \in N x$, then there exists $f \in S_{F, x}$ such that for each $t \in J$, we have

$$
\begin{aligned}
u(t)= & C(t)\left[\phi(0)-h_{0}(x)\right]+S(t)\left[y_{0}-g\left(0, x_{0}\right)\right] \\
& +\int_{0}^{t} C(t-s) g\left(s, x_{s}\right) d s+\int_{0}^{t} S(t-s) f(s) d s .
\end{aligned}
$$

By (H4)-(H6), we have, for each $t \in J$,

$$
\begin{aligned}
|u(t)| \leq & \left|C(t)\left[\phi(0)-h_{0}(x)\right]\right|+\left|S(t)\left[y_{0}-g\left(0, x_{0}\right)\right]\right| \\
& +\left|\int_{0}^{t} C(t-s) g\left(s, x_{s}\right) d s\right|+\left|\int_{0}^{t} S(t-s) f(s) d s\right| \\
\leq & M(\|\phi\|+G)+M T\left[\left|y_{0}\right|+c_{1}|| x_{0} \|+2 c_{2}\right] \\
& +M c_{1} \int_{0}^{t}\left\|x_{s}\right\| d s+M T \sup _{x \in[0, q]} \psi(2 x)\left(\int_{0}^{t} p(s) d s\right) .
\end{aligned}
$$


By

$$
\begin{aligned}
u^{\prime}(t)= & A S(t)\left[\phi(0)-h_{0}(x)\right]+C(t)\left[y_{0}-g\left(0, x_{0}\right)\right]+g\left(t, x_{t}\right) \\
& +\int_{0}^{t} A S(t-s) g\left(s, x_{s}\right) d s+\int_{0}^{t} C(t-s) f(s) d s,
\end{aligned}
$$

we have for each $t \in J$,

$$
\begin{aligned}
\left|u^{\prime}(t)\right| \leq & \left|A S(t)\left[\phi(0)-h_{0}(x)\right]\right|+\left|C(t)\left[y_{0}-g\left(0, x_{0}\right)\right]\right|+\left|g\left(t, x_{t}\right)\right| \\
& +\left|\int_{0}^{t} A S(t-s) g\left(s, x_{s}\right) d s\right|+\left|\int_{0}^{t} C(t-s) f(s) d s\right| \\
\leq & M^{*}(\|\phi\|+G)+M\left[\left|y_{0}\right|+c_{1}\left\|x_{0}\right\|+c_{2}\right]+c_{1}\left\|x_{t}\right\|+c_{2}+M^{*} T c_{2} \\
& +M^{*} c_{1} \int_{0}^{t}\left\|x_{s}\right\| d s+M \sup _{x \in[0, q]} \psi(2 x)\left(\int_{0}^{t} p(s) d s\right) .
\end{aligned}
$$

Then for each $h \in N\left(B_{q}\right)$, we have

$$
\begin{aligned}
\|u\|^{*} \leq & \left(M+M^{*}\right)(\|\phi\|+G)+M(1+T)\left(\left|y_{0}\right|+c_{1}\left\|x_{0}\right\|+c_{2}\right) \\
& +\left(c_{1} q+c_{2}\right)\left(1+M T+M^{*} T\right)+M(1+T) \sup _{x \in[0, q]} \psi(2 x)\left(\int_{0}^{T} p(s) d s\right):=\ell .
\end{aligned}
$$

STEP 3. $N$ maps bounded sets into equicontinuous sets of $Z$.

Let $t_{1}, t_{2} \in J, 0<t_{1}<t_{2}$, and let $B_{q}=\left\{x \in Z:\|x\|^{*} \leq q\right\}$ be a bounded set of $C\left(J_{1}, X\right)$. For each $x \in B_{q}$ and $u \in N x$, there exists $f \in S_{F, x}$ such that for $t \in J$, (3.17) holds. Thus,

$$
\begin{aligned}
\left|u\left(t_{1}\right)-u\left(t_{2}\right)\right| \leq & \left|\left[C\left(t_{1}\right)-C\left(t_{2}\right)\right]\left[\phi(0)-h_{0}(x)\right]\right|+\left|\left[S\left(t_{1}\right)-S\left(t_{2}\right)\right]\left[y_{0}-g\left(0, x_{0}\right)\right]\right| \\
& +\left|\int_{0}^{t_{1}}\left[C\left(t_{1}-s\right)-C\left(t_{2}-s\right)\right] g\left(s, x_{s}\right) d s\right|+\left|\int_{t_{1}}^{t_{2}} C\left(t_{2}-s\right) g\left(s, x_{s}\right) d s\right| \\
& +\left|\int_{0}^{t_{1}}\left[S\left(t_{1}-s\right)-S\left(t_{2}-s\right)\right] f(s) d s\right|+\left|\int_{t_{1}}^{t_{2}} S\left(t_{2}-s\right) f(s) d s\right| \\
\leq & \left|C\left(t_{1}\right)-C\left(t_{2}\right)\right|(\|\phi\|+G)+\left|S\left(t_{1}\right)-S\left(t_{2}\right)\right|\left[\left|y_{0}\right|+c_{1}|| x_{0}||+c_{2}\right] \\
& +\int_{0}^{t_{1}}\left|C\left(t_{1}-s\right)-C\left(t_{2}-s\right)\right|\left[c_{1}|| x_{s}||+c_{2}\right] d s \\
& +\int_{t_{1}}^{t_{2}}\left|C\left(t_{2}-s\right)\right|\left[c_{1}|| x_{s}||+c_{2}\right] d s \\
& +\int_{0}^{t_{1}}\left|S\left(t_{1}-s\right)-S\left(t_{2}-s\right)\right||f(s)| d s+\int_{t_{1}}^{t_{2}}\left|S\left(t_{2}-s\right)\right||f(s)| d s,
\end{aligned}
$$


and similarly,

$$
\begin{aligned}
\left|u^{\prime}\left(t_{1}\right)-u^{\prime}\left(t_{2}\right)\right| \leq & \left|A\left[S\left(t_{1}\right)-S\left(t_{2}\right)\right]\left[\phi(0)-h_{0}(x)\right]\right| \\
& +\left|\left[C\left(t_{1}\right)-C\left(t_{2}\right)\right]\left[y_{0}-g\left(0, x_{0}\right)\right]\right|+\left|g\left(t_{1}, x_{t_{1}}\right)-g\left(t_{2}, x_{t_{2}}\right)\right| \\
& +\left|\int_{0}^{t_{1}} A\left[S\left(t_{1}-s\right)-S\left(t_{2}-s\right)\right] g\left(s, x_{s}\right) d s\right| \\
& +\left|\int_{t_{1}}^{t_{2}} A S\left(t_{2}-s\right) g\left(s, x_{s}\right) d s\right|+\left|\int_{0}^{t_{1}}\left[C\left(t_{1}-s\right)-C\left(t_{2}-s\right)\right] f(s) d s\right| \\
& +\left|\int_{t_{1}}^{t_{2}} C\left(t_{2}-s\right) f(s) d s\right| \\
\leq & \left|A\left[S\left(t_{1}\right)-S\left(t_{2}\right)\right]\right|(\|\phi\|+G) \\
& +\left|S\left(t_{1}\right)-S\left(t_{2}\right)\right|\left[\left|y_{0}\right|+c_{1}|| x_{0}||+c_{2}\right]+\left|g\left(t_{1}, x_{t_{1}}\right)-g\left(t_{2}, x_{t_{2}}\right)\right| \\
& +\int_{0}^{t_{1}}\left|A\left[S\left(t_{1}-s\right)-S\left(t_{2}-s\right)\right]\right|\left[c_{1}|| x_{s}||+c_{2}\right] d s \\
& +\int_{t_{1}}^{t_{2}}\left|A S\left(t_{2}-s\right)\right|\left[c_{1}|| x_{s} \|+c_{2}\right] d s \\
& +\int_{0}^{t_{1}}\left|C\left(t_{1}-s\right)-C\left(t_{2}-s\right)\right||f(s)| d s+\int_{t_{1}}^{t_{2}}\left|C\left(t_{2}-s\right)\right||f(s)| d s .
\end{aligned}
$$

The right-hand side of the above inequalities tend to zero as $t_{2}-t_{1} \rightarrow 0$, since $C(t)$, $S(t)$ are uniformly continuous for $t \in J$ and the compactness of $C(t), S(t)$ for $t>0$ imply the continuity in the uniform operator topology. The compactness of $S(t)$ follows from that of $C(t)$ (see [29]).

The equicontinuity for the cases $t_{1}<t_{2} \leq 0$ and $t_{1} \leq 0 \leq t_{2}$ is obvious. As a consequence of Steps 2 and 3, (H2), (H4), and (H5) together with the Ascoli-Arzela theorem, we can conclude that $N: Z \rightarrow 2^{Z}$ is a completely continuous multivalued map, and therefore, a condensing map.

STEP 4. $N$ has a closed graph.

Let $x_{n} \rightarrow x_{*}, u_{n} \in N x_{n}$, and $u_{n} \rightarrow u_{*}$. We will prove that $u_{*} \in N x_{*}$. Clearly, $x_{n}^{\prime} \rightarrow x_{*}^{\prime}$, $u_{n}^{\prime} \in N x_{n}^{\prime}$, and $u_{n}^{\prime} \rightarrow u_{*}^{\prime} . u_{n} \in N x_{n}$ means that there exists $f_{n} \in S_{F, x_{n}}$ such that for $t \in J$

$$
\begin{aligned}
u_{n}(t)= & C(t)\left[\phi(0)-h_{0}\left(x_{n}\right)\right]+S(t)\left[y_{0}-g\left(0, x_{n 0}\right)\right]+\int_{0}^{t} C(t-s) g\left(s, x_{n s}\right) d s \\
& +\int_{0}^{t} S(t-s) f_{n}(s) d s .
\end{aligned}
$$

We must prove that there exists $f_{*} \in S_{F, x_{*}}$ such that for $t \in J$,

$$
\begin{aligned}
u_{*}(t)= & C(t)\left[\phi(0)-h_{0}\left(x_{*}\right)\right]+S(t)\left[y_{0}-g\left(0, x_{* 0}\right)\right]+\int_{0}^{t} C(t-s) g\left(s, x_{* s}\right) d s \\
& +\int_{0}^{t} S(t-s) f_{*}(s) d s .
\end{aligned}
$$


Clearly, we have that, as $n \rightarrow \infty$,

$$
\begin{aligned}
& \mid\left(u_{n}(t)-C(t)\left(\phi(0)-h_{0}\left(x_{n}\right)\right)-S(t)\left(y_{0}-g\left(0, x_{n 0}\right)\right)-\int_{0}^{t} C(t-s) g\left(s, x_{n s}\right) d s\right) \\
& -\left(u_{*}(t)-C(t)\left(\phi(0)-h_{0}\left(x_{*}\right)\right)-S(t)\left(y_{0}-g\left(0, x_{* 0}\right)\right)-\int_{0}^{t} C(t-s) g\left(s, x_{* s}\right) d s\right) \mid \rightarrow 0, \\
& \mid\left(u_{n}^{\prime}(t)-A S(t)\left(\phi(0)-h_{0}\left(x_{n}\right)\right)-C(t)\left(y_{0}-g\left(0, x_{n 0}\right)\right)-g\left(t, x_{n t}\right)-\int_{0}^{t} A S(t-s) g\left(s, x_{n s}\right) d s\right) \\
& \quad-\left(u_{*}^{\prime}(t)-A S(t)\left(\phi(0)-h_{0}\left(x_{*}\right)\right)-C(t)\left(y_{0}-g\left(0, x_{* 0}\right)\right)\right. \\
& \left.\quad-g\left(t, x_{* t}\right)-\int_{0}^{t} A S(t-s) g\left(s, x_{* s}\right) d s\right) \mid \rightarrow 0 .
\end{aligned}
$$

Consider the linear and continuous operator $\Gamma: L^{1}(J, X) \rightarrow C(J, X)$ defined as

$$
f \rightarrow \Gamma(f)(t)=\int_{0}^{t} S(t-s) f(s) d s .
$$

From Lemma 3.3, it follows that $\Gamma \circ S_{F}$ is a closed graph operator. Moreover, we have that

$$
u_{n}(t)-C(t)\left[\phi(0)-h_{0}(x)\right]-S(t)\left[y_{0}-g\left(0, x_{0}\right)\right]-\int_{0}^{t} C(t-s) g\left(s, x_{n s}\right) d s \in \Gamma\left(S_{F, x_{n}}\right) .
$$

Since $x_{n} \rightarrow x_{*}$, it follows from Lemma 3.3 that

$$
\begin{aligned}
u_{*}(t) & -C(t)\left[\phi(0)-h_{0}(x)\right]-S(t)\left[y_{0}-g\left(0, x_{0}\right)\right]-\int_{0}^{T} C(t-s) g\left(s, x_{* s}\right) d s \\
= & \int_{0}^{t} S(t-s) f_{*}(s) d s
\end{aligned}
$$

for some $f_{*} \in S_{F, x_{*}}$. Therefore, $N$ is a completely continuous multivalued map, upper semicontinuous with convex closed values.

STEP 5. The set

$$
\Omega:=\{x \in Z: \lambda x \in N x \text {, for some } \lambda>1\}
$$

is bounded.

Let $x \in \Omega$. Then $\lambda x \in N x$ for some $\lambda>1$. Thus, there exists $f \in S_{F, x}$ such that

$$
\begin{aligned}
x(t)= & \lambda^{-1} C(t)\left[\phi(0)-h_{0}(x)\right]+\lambda^{-1} S(t)\left[y_{0}-g\left(0, x_{0}\right)\right] \\
& +\lambda^{-1} \int_{0}^{t} C(t-s) g\left(s, x_{s}\right) d s+\lambda^{-1} \int_{0}^{t} S(t-s) f(s) d s, \quad t \in J .
\end{aligned}
$$

This implies by (H5)-(H6) that for each $t \in J$, we have

$$
\begin{aligned}
|x(t)| \leq & M(\|\phi\|+G)+M T\left[\left|y_{0}\right|+c_{1}|| x_{0} \|+2 c_{2}\right] \\
& +M c_{1} \int_{0}^{t}\left\|x_{s}\right\| d s+M T \int_{0}^{t} p(s) \psi\left(\left\|x_{s}\right\|+\left|x^{\prime}(s)\right|\right) d s .
\end{aligned}
$$


We consider the function

$$
\mu(t)=\sup \{|x(s)|:-r \leq s \leq t\}, \quad t \in J .
$$

Let $t^{*} \in[-r, t]$ be such that $\mu(t)=\left|x\left(t^{*}\right)\right|$. If $t^{*} \in J_{0}$, then $\mu(t) \leq\|\phi\|+G$.

If $t^{*} \in J$, we have for $t \in J$,

$$
\begin{aligned}
\mu(t) \leq & M(\|\phi\|+G)+M T\left[\left|y_{0}\right|+c_{1}(\|\phi\|+G)+2 c_{2}\right]+M c_{1} \int_{0}^{t^{*}} \mu(s) d s \\
& +M T \int_{0}^{t^{*}} p(s) \psi\left(\mu(s)+\left|x^{\prime}(s)\right|\right) d s \\
\leq & M(\|\phi\|+G)+M T\left[\left|y_{0}\right|+c_{1}(\|\phi\|+G)+2 c_{2}\right]+M c_{1} \int_{0}^{t} \mu(s) d s \\
& +M T \int_{0}^{t} p(s) \psi\left(\mu(s)+\left|x^{\prime}(s)\right|\right) d s .
\end{aligned}
$$

Denoting by $q(t)$ the right-hand side of the above inequality, we have

$$
\begin{aligned}
& q(0)=M(\|\phi\|+G)+M T\left[\left|y_{0}\right|+c_{1}(\|\phi\|+G)+2 c_{2}\right], \\
& \mu(t) \leq q(t), \quad t \in J, \\
& q^{\prime}(t)=M c_{1} \mu(t)+M T p(t) \psi\left(\mu(t)+\left|x^{\prime}(t)\right|\right), \quad t \in J .
\end{aligned}
$$

By

$$
\begin{aligned}
x^{\prime}(t)= & \lambda^{-1} A S(t)\left[\phi(0)-h_{0}(x)\right]+\lambda^{-1} C(t)\left[y_{0}-g\left(0, x_{0}\right)\right]+\lambda^{-1} g\left(t, x_{t}\right) \\
& +\lambda^{-1} \int_{0}^{t} A S(t-s) g\left(s, x_{s}\right) d s+\lambda^{-1} \int_{0}^{t} C(t-s) f(s) d s, \quad t \in J,
\end{aligned}
$$

we obtain

$$
\begin{aligned}
\left|x^{\prime}(t)\right| \leq & M^{*}(\|\phi\|+G)+M\left[\left|y_{0}\right|+c_{1}\left\|x_{0}\right\|+c_{2}\right]+M^{*} c_{2} T+c_{1}\left\|x_{t}\right\|+c_{2} \\
& +M^{*} c_{1} \int_{0}^{t}\left\|x_{s}\right\| d s+M \int_{0}^{t} p(s) \psi\left(\left\|x_{s}\right\|+\left|x^{\prime}(s)\right|\right) d s .
\end{aligned}
$$

Let

$$
\gamma(t)=\sup \left\{\left|x^{\prime}(s)\right|: 0 \leq s \leq t\right\}, \quad t \in J .
$$

Let $t^{*} \in[0, t]$ be such that $\gamma(t)=\left|x^{\prime}\left(t^{*}\right)\right|$. By the previous inequality, we have for $t \in J$,

$$
\begin{aligned}
\gamma(t) \leq & M^{*}(\|\phi\|+G)+M\left[\left|y_{0}\right|+c_{1}(\|\phi\|+G)+c_{2}\right]+M^{*} c_{2} T+c_{1}\left\|x_{t^{*}}\right\|+c_{2} \\
& +M^{*} c_{1} \int_{0}^{t^{*}}\left\|x_{s}\right\| d s+M \int_{0}^{t^{*}} p(s) \psi\left(\left\|x_{s}\right\|+\gamma(s)\right) d s \\
\leq & M^{*}(\|\phi\|+G)+M\left[\left|y_{0}\right|+c_{1}(\|\phi\|+G)+c_{2}\right]+M^{*} c_{2} T+c_{1} q(t)+c_{2} \\
& +M^{*} c_{1} \int_{0}^{t} q(s) d s+M \int_{0}^{t} p(s) \psi(q(s)+\gamma(s)) d s .
\end{aligned}
$$


Denoting by $r(t)$ the right-hand side of the above inequality, we have

$$
\begin{gathered}
r(0)=M^{*}(\|\phi\|+G)+M\left[\left|y_{0}\right|+c_{1}(\|\phi\|+G)+c_{2}\right]+M^{*} c_{2} T+c_{1} q(0)+c_{2}, \\
\quad \gamma(t) \leq r(t), \quad t \in J, \\
r^{\prime}(t)=M^{*} c_{1} q(t)+c_{1} q^{\prime}(t)+M p(t) \psi(q(t)+\gamma(t)), \quad t \in J .
\end{gathered}
$$

Let $\omega(t)=q(t)+r(t), t \in J$. Then $\omega(0)=c$ and for $t \in J$,

$$
\begin{aligned}
\omega^{\prime}(t)= & q^{\prime}(t)+r^{\prime}(t) \\
\leq & \left(1+c_{1}\right)\left[M c_{1} q(t)+M T p(t) \psi(q(t)+r(t))\right] \\
& +M^{*} c_{1} q(t)+M p(t) \psi(q(t)+r(t)) \\
\leq & \left(M c_{1}+M^{*} c_{1}+M c_{1}^{2}\right) \omega(t)+\left(M+M T+M T c_{1}\right) p(t) \psi \omega(t) \\
\leq & m(t) \omega(t)+\psi(\omega(t)) .
\end{aligned}
$$

This implies that for each $t \in J$,

$$
\int_{\omega(0)}^{\omega(t)} \frac{d s}{s+\psi(s)} \leq \int_{0}^{T} m(s) d s<\int_{\omega(0)}^{\infty} \frac{d s}{s+\psi(s)} .
$$

This inequality implies that there exists a constant $L$ such that $\omega(t) \leq L, t \in J$. Then

$$
\begin{aligned}
|x(t)| \leq \mu(t) \leq q(t) \leq L, & t \in J_{1}, \\
\left|x^{\prime}(t)\right| \leq \gamma(t) \leq r(t) \leq L, & t \in J,
\end{aligned}
$$

and hence

$$
\|x\|^{*}=\max \left\{\|x\|_{J_{1}},\left\|x^{\prime}\right\|_{J}\right\} \leq L,
$$

where $L$ depends only on $T$ and on the function $p$ and $\psi$. This shows that $\Omega$ is bounded.

As a consequence of Lemma 2.3, we deduce that $N$ has a fixed point which is a mild solution of the system (1.1).

4. Second-order neutral integrodifferential inclusions. In this section, we study the existence problem (1.5). We need the following assumptions.

(H7) For each $t \in J, K(t, s)$ is measurable on $[0, t]$ and

$$
H(t)=\operatorname{ess} \sup \{|K(t, s)|, 0 \leq s \leq t\}
$$

is bounded on $J$.

(H8) The map $t \rightarrow K_{t}$ is continuous from $J$ to $L^{\infty}(J, \mathbb{R})$; here $K_{t}(s)=K(t, s)$.

(H9) $\|F(t, u, v)\|:=\sup \{|w|: w \in F(t, u, v)\} \leq p(t) \psi(\|u\|+|v|)$ for almost all $t \in J$, $u \in C\left(J_{0}, X\right)$, and $v \in X$, where $p \in L^{1}\left(J, \mathbb{R}_{+}\right)$and $\psi: \mathbb{R}_{+} \rightarrow(0, \infty)$ is continuous and increasing with

$$
\int_{0}^{T} m(s) d s<\int_{c}^{\infty} \frac{d s}{s+\psi(s)}<\infty,
$$


where

$$
m^{*}(t)=\max \left\{M c_{1}+M c_{1}^{2}+M^{*} c_{1},\left(M T+M T^{2}+M T^{2} c_{1}\right) \sup _{t \in J} H(t) p(t)\right\} .
$$

We define the mild solution for the problem (1.5) by the integral equation

$$
\begin{aligned}
x(t)= & C(t)\left[\phi(0)-h_{0}(x)\right]+S(t)\left[y_{0}-g\left(0, x_{0}\right)\right]+\int_{0}^{t} C(t-s) g\left(s, x_{s}\right) d s \\
& +\int_{0}^{t} S(t-s) \int_{0}^{s} K(s, u) f(u) d u d s, \quad t \in J,
\end{aligned}
$$

where $f \in S_{F, x}=\left\{f \in L^{1}(J, X): f(t) \in F\left(t, x_{t}, x^{\prime}(t)\right)\right.$ for a.e. $\left.t \in J\right\}$.

Definition 4.1. A function $x:[-r, T] \rightarrow X, T>0$, is called a mild solution of the problem (1.5) if $x(t)+h_{t}(x)=\phi(t), t \in[-r, 0]$, and there exists a $v \in L^{1}(J, X)$ such that $v(t) \in F\left(t, x_{t}, x^{\prime}(t)\right)$ a.e. on $J$, and the integral equation (4.4) is satisfied.

THEOREM 4.2. Assume that hypotheses (H1)-(H5), (H7)-(H9) are satisfied. Then system (1.5) has at least one mild solution on $J_{1}$.

Proof. Consider the multivalued map $Q: Z \rightarrow 2^{Z}$ defined by $Q x$, the set of functions $u \in Z$ such that

$$
u(t)= \begin{cases}\phi(t)-h_{t}(x) & \text { if } t \in J_{0}, \\ C(t)\left[\phi(0)-h_{0}(x)\right]+S(t)\left[y_{0}-g\left(0, x_{0}\right)\right]+\int_{0}^{t} C(t-s) g\left(s, x_{s}\right) d s & \\ +\int_{0}^{t} S(t-s) \int_{0}^{s} K(s, \tau) f(\tau) d \tau d s & \text { if } t \in J,\end{cases}
$$

where (3.9) holds. We remark that the fixed points of $Q$ are mild solutions to (1.5).

As in Theorem 4.2, with appropriate modifications we can easily show that $Q$ is a completely continuous multivalued map, upper semicontinuous with convex closed values. Here we repeat only Step 5 . That is, we prove that the set

$$
\Omega:=\{x \in Z: \lambda x \in Q x \text {, for some } \lambda>1\}
$$

is bounded.

Let $x \in \Omega$. Then $\lambda x \in Q x$ for some $\lambda>1$. Thus, there exists $f \in S_{F, x}$ such that

$$
\begin{aligned}
x(t)= & \lambda^{-1} C(t)\left[\phi(0)-h_{0}(x)\right]+\lambda^{-1} S(t)\left[y_{0}-g\left(0, x_{0}\right)\right]+\lambda^{-1} \int_{0}^{t} C(t-s) g\left(s, x_{s}\right) d s \\
& +\lambda^{-1} \int_{0}^{t} S(t-s) \int_{0}^{s} K(s, \tau) f(\tau) d \tau d s, \quad t \in J .
\end{aligned}
$$


This implies by (H4)-(H9) that for each $t \in J$, we have

$$
\begin{aligned}
|x(t)| \leq & M(\|\phi\|+G)+M T\left[\left|y_{0}\right|+c_{1}|| x_{0} \|+2 c_{2}\right] \\
& +M c_{1} \int_{0}^{t}\left\|x_{s}\right\| d s+M T^{2} \sup _{t \in J} H(t) \int_{0}^{t} p(s) \psi\left(\left\|x_{s}\right\|+\left|x^{\prime}(s)\right|\right) d s .
\end{aligned}
$$

We consider the function (3.33). Let $t^{*} \in[-r, t]$ be such that $\mu(t)=\left|x\left(t^{*}\right)\right|$. If $t^{*} \in J_{0}$, then $\mu(t) \leq\|\phi\|+G$.

If $t^{*} \in J$, by the previous inequality we have for $t \in J$,

$$
\begin{aligned}
\mu(t) \leq & M(\|\phi\|+G)+M T\left[\left|y_{0}\right|+c_{1}(\|\phi\|+G)+2 c_{2}\right] \\
& +M c_{1} \int_{0}^{t^{*}}\left\|x_{s}\right\| d s+M T^{2} \sup _{t \in J} H(t) \int_{0}^{t^{*}} p(s) \psi\left(\left\|x_{s}\right\|+\left|x^{\prime}(s)\right|\right) d s \\
\leq & M(\|\phi\|+G)+M T\left[\left|y_{0}\right|+c_{1}(\|\phi\|+G)+2 c_{2}\right] \\
& +M c_{1} \int_{0}^{t} \mu(s) d s+M T^{2} \sup _{t \in J} H(t) \int_{0}^{t} p(s) \psi\left(\mu(s)+\left|x^{\prime}(s)\right|\right) d s .
\end{aligned}
$$

We denote the right-hand side of the above inequality as $q_{1}(t)$. Then, we have

$$
\begin{gathered}
q_{1}(0)=M(\|\phi\|+G)+M T\left[\left|y_{0}\right|+c_{1}(\|\phi\|+G)+2 c_{2}\right], \\
\mu(t) \leq q_{1}(t), \quad t \in J, \\
q_{1}^{\prime}(t)=M c_{1} \mu(t)+M T^{2} \sup _{t \in J} H(t) p(t) \psi\left(\mu(t)+\left|x^{\prime}(t)\right|\right), \quad t \in J .
\end{gathered}
$$

By

$$
\begin{aligned}
x^{\prime}(t)= & \lambda^{-1} A S(t)\left[\phi(0)-h_{0}(x)\right]+\lambda^{-1} C(t)\left[y_{0}-g\left(0, x_{0}\right)\right]+\lambda^{-1} g\left(t, x_{t}\right) \\
& +\lambda^{-1} \int_{0}^{t} A S(t-s) g\left(s, x_{s}\right) d s+\lambda^{-1} \int_{0}^{t} C(t-s) \int_{0}^{s} K(s, \tau) f(\tau) d \tau d s, \quad t \in J,
\end{aligned}
$$

we have

$$
\begin{aligned}
\left|x^{\prime}(t)\right| \leq & M^{*}(\|\phi\|+G)+M\left[\left|y_{0}\right|+c_{1}\left\|x_{0}\right\|+c_{2}\right]+c_{1}\left\|x_{t}\right\|+c_{2}+M^{*} T c_{2} \\
& +M^{*} c_{1} \int_{0}^{t}\left\|x_{s}\right\| d s+M T \sup _{t \in J} H(t) \int_{0}^{t} p(s) \psi\left(\left\|x_{s}\right\|+\left|x^{\prime}(s)\right|\right) d s .
\end{aligned}
$$

Let (3.38) holds.

Let $t^{*} \in[0, t]$ be such that $\gamma(t)=\left|x\left(t^{*}\right)\right|$. By the previous inequality, we have for $t \in J$,

$$
\begin{aligned}
\gamma(t) \leq & M^{*}(\|\phi\|+G)+M\left[\left|y_{0}\right|+c_{1}(\|\phi\|+G)+c_{2}\right]+c_{1}\left\|x_{t^{*}}\right\|+c_{2}+M^{*} T c_{2} \\
& +M^{*} c_{1} \int_{0}^{t^{*}}\left\|x_{s}\right\| d s+M T \sup _{t \in J} H(t) \int_{0}^{t^{*}} p(s) \psi\left(\left\|x_{s}\right\|+\left|x^{\prime}(s)\right|\right) d s \\
\leq & M(\|\phi\|+G)+M T\left[\left|y_{0}\right|+c_{1}(\|\phi\|+G)+c_{2}\right]+c_{1} q_{1}(t)+c_{2}+M^{*} T c_{2} \\
& +M^{*} c_{1} \int_{0}^{t} q_{1}(s) d s+M T \sup _{t \in J} H(t) \int_{0}^{t} p(s) \psi\left(q_{1}(s)+\gamma(s)\right) d s .
\end{aligned}
$$


We denote the right-hand side of the above inequality as $r_{1}(t)$. Then, we have

$$
\begin{gathered}
r_{1}(0)=M^{*}(\|\phi\|+G)+M\left[\left|y_{0}\right|+c_{1}(\|\phi\|+G)+c_{2}\right]+c_{1} q_{1}(0)+c_{2}+M^{*} T c_{2}, \\
\gamma(t) \leq r_{1}(t), \quad t \in J, \\
r_{1}^{\prime}(t)=M^{*} c_{1} q_{1}(t)+c_{1} q_{1}^{\prime}(t)+M T \sup _{t \in J} H(t) p(t) \psi\left(q_{1}(t)+\gamma(t)\right), \quad t \in J .
\end{gathered}
$$

Let $\omega_{1}(t)=q_{1}(t)+r_{1}(t), t \in J$. Then $\omega_{1}(0)=c$ and for $t \in J$,

$$
\begin{aligned}
\omega_{1}^{\prime}(t)= & q_{1}^{\prime}(t)+r_{1}^{\prime}(t) \\
\leq & \left(1+c_{1}\right)\left[M c_{1} q_{1}(t)+M T^{2} \sup _{t \in J} H(t) p(t) \psi\left(q_{1}(t)+r_{1}(t)\right)\right] \\
& +M^{*} c_{1} q_{1}(t)+M T \sup _{t \in J} H(t) p(t) \psi\left(q_{1}(t)+r_{1}(t)\right) \\
\leq & \left(M c_{1}+M^{*} c_{1}+M c_{1}^{2}\right) \omega_{1}(t)+\left(M T+M T^{2}+M T^{2} c_{1}\right) \sup _{t \in J} H(t) p(t) \psi \omega_{1}(t) \\
\leq & m^{*}(t) \omega_{1}(t)+\psi\left(\omega_{1}(t)\right) .
\end{aligned}
$$

This implies that for each $t \in J$,

$$
\int_{\omega_{1}(0)}^{\omega_{1}(t)} \frac{d s}{s+\psi(s)} \leq \int_{0}^{T} m^{*}(s) d s<\int_{c}^{\infty} \frac{d s}{s+\psi(s)} .
$$

This inequality implies that there exists a constant $L$ such that $\omega_{1}(t) \leq L, t \in J$. Then

$$
\begin{aligned}
|x(t)| \leq \mu(t) \leq q_{1}(t) \leq L, & t \in J_{1}, \\
\left|x^{\prime}(t)\right| \leq \gamma(t) \leq r_{1}(t) \leq L, & t \in J,
\end{aligned}
$$

and hence (3.44) holds, where $L$ depends only on $T$ and on the function $p$ and $\psi$. This shows that $\Omega$ is bounded.

As a consequence of Lemma 2.3, we deduce that $Q$ has a fixed point and thus system (1.1) has at least one mild solution on $J_{1}$.

ACKNOWLEDGMENT. The work of the first and second authors was supported by Brain Korea 21 project at Yonsei University, 2003.

\section{REFERENCES}

[1] E. P. Avgerinos and N. S. Papageorgiou, On quasilinear evolution inclusions, Glas. Mat. Ser. III 28 (1993), no. 1, 35-52.

[2] K. Balachandran and M. Chandrasekaran, Existence of solutions of a delay differential equation with nonlocal condition, Indian J. Pure Appl. Math. 27 (1996), no. 5, 443-449.

[3] _ The non-local Cauchy problem for semilinear integrodifferential equations with deviating argument, Proc. Edinb. Math. Soc. (2) 44 (2001), no. 1, 63-70.

[4] J. M. Ball, Initial-boundary value problems for an extensible beam, J. Math. Anal. Appl. 42 (1973), 61-90.

[5] J. Banaś and K. Goebel, Measures of Noncompactness in Banach Spaces, Lecture Notes in Pure and Applied Mathematics, vol. 60, Marcel Dekker, New York, 1980. 
[6] M. Benchohra and S. K. Ntouyas, An existence result on noncompact intervals for second order functional differential inclusions, J. Math. Anal. Appl. 248 (2000), no. 2, 520531.

[7] __ Nonlocal Cauchy problems for neutral functional differential and integrodifferential inclusions in Banach spaces, J. Math. Anal. Appl. 258 (2001), no. 2, 573-590.

[8] J. Bochenek, An abstract nonlinear second order differential equation, Ann. Polon. Math. 54 (1991), no. 2, 155-166.

[9] L. Byszewski, Theorem about existence and uniqueness of continuous solution of nonlocal problem for nonlinear hyperbolic equation, Appl. Anal. 40 (1991), no. 2-3, 173-180.

[10] - Theorems about the existence and uniqueness of solutions of a semilinear evolution nonlocal Cauchy problem, J. Math. Anal. Appl. 162 (1991), no. 2, 494-505.

[11] L. Byszewski and V. Lakshmikantham, Theorem about the existence and uniqueness of a solution of a nonlocal abstract Cauchy problem in a Banach space, Appl. Anal. 40 (1991), no. 1, 11-19.

[12] J. P. Dauer and K. Balachandran, Existence of solutions for an integrodifferential equation with nonlocal condition in Banach spaces, Libertas Math. 16 (1996), 133-143.

[13] K. Deimling, Multivalued Differential Equations, de Gruyter Series in Nonlinear Analysis and Applications, vol. 1, Walter de Gruyter, Berlin, 1992.

[14] K. Deng, Exponential decay of solutions of semilinear parabolic equations with nonlocal initial conditions, J. Math. Anal. Appl. 179 (1993), no. 2, 630-637.

[15] H. O. Fattorini, Ordinary differential equations in linear topological spaces. I, J. Differential Equations 5 (1969), 72-105.

[16] _ _ Ordinary differential equations in linear topological spaces. II, J. Differential Equations 6 (1969), 50-70.

[17] W. E. Fitzgibbon, Global existence and boundedness of solutions to the extensible beam equation, SIAM J. Math. Anal. 13 (1982), no. 5, 739-745.

[18] J. A. Goldstein, Semigroups of Linear Operators and Applications, Oxford Mathematical Monographs, Oxford University Press, New York, 1985.

[19] E. Hernández and H. R. Henríquez, Existence of periodic solutions of partial neutral functional-differential equations with unbounded delay, J. Math. Anal. Appl. 221 (1998), no. 2, 499-522.

[20] _ Existence results for partial neutral functional-differential equations with unbounded delay, J. Math. Anal. Appl. 221 (1998), no. 2, 452-475.

[21] S. Hu and N. S. Papageorgiou, Handbook of Multivalued Analysis. Vol. I, Mathematics and Its Applications, vol. 419, Kluwer Academic Publishers, Dordrecht, 1997.

[22] A. Lasota and Z. Opial, An application of the Kakutani-Ky Fan theorem in the theory of ordinary differential equations, Bull. Acad. Polon. Sci. Sér. Sci. Math. Astronom. Phys. 13 (1965), 781-786.

[23] Y. Lin and J. H. Liu, Semilinear integrodifferential equations with nonlocal Cauchy problem, Nonlinear Anal. 26 (1996), no. 5, 1023-1033.

[24] M. Martelli, A Rothe's type theorem for non-compact acyclic-valued maps, Boll. Un. Mat. Ital. (4) 11 (1975), no. 3, 70-76.

[25] S. K. Ntouyas and P. Ch. Tsamatos, Global existence for second order semilinear ordinary and delay integrodifferential equations with nonlocal conditions, Appl. Anal. 67 (1997), no. 3-4, 245-257.

[26] Global existence for semilinear evolution equations with nonlocal conditions, J. Math. Anal. Appl. 210 (1997), no. 2, 679-687.

[27] N. S. Papageorgiou, Boundary value problems for evolution inclusions, Comment. Math. Univ. Carolin. 29 (1988), no. 2, 355-363.

[28] _ Mild solutions of semilinear evolution inclusions and optimal control, Indian J. Pure Appl. Math. 26 (1995), no. 3, 189-216.

[29] C. C. Travis and G. F. Webb, Compactness, regularity, and uniform continuity properties of strongly continuous cosine families, Houston J. Math. 3 (1977), no. 4, 555-567. 
Cosine families and abstract nonlinear second order differential equations, Acta Math. Acad. Sci. Hungar. 32 (1978), no. 1-2, 75-96.

[31] _ Second order differential equations in Banach space, Nonlinear Equations in Abstract Spaces (Proc. Internat. Sympos., Univ. Texas, Arlington, Tex, 1977), Academic Press, New York, 1978, pp. 331-361.

[32] K. Yosida, Functional Analysis, 6th ed., Grundlehren der Mathematischen Wissenschaften, vol. 123, Springer-Verlag, Berlin, 1980.

S. Marshal Anthoni: Department of Mathematics, Yonsei University, Seoul 120-749, Korea E-mail address: smanthony@ycos.com

J.-H. Kim: Department of Mathematics, Yonsei University, Seoul 120-749, Korea

E-mail address: jhkim96@yonsei .ac.kr

J. P. Dauer: Department of Mathematics, University of Tennessee at Chattanooga, Chattanooga, TN 37403-2598, USA

E-mail address: jera1d-dauer@utc.edu 


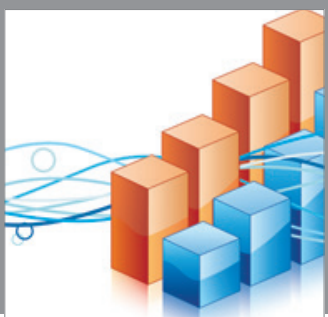

Advances in

Operations Research

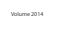

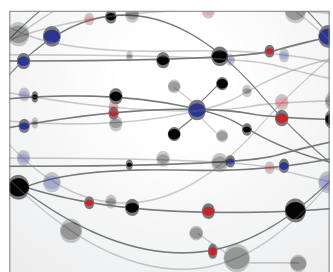

\section{The Scientific} World Journal
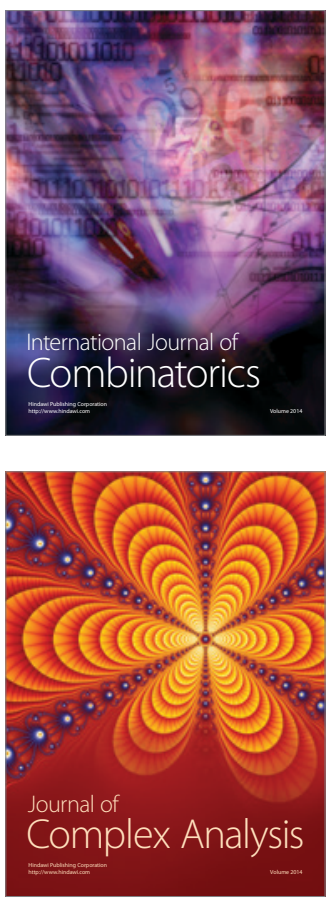

International Journal of

Mathematics and

Mathematical

Sciences
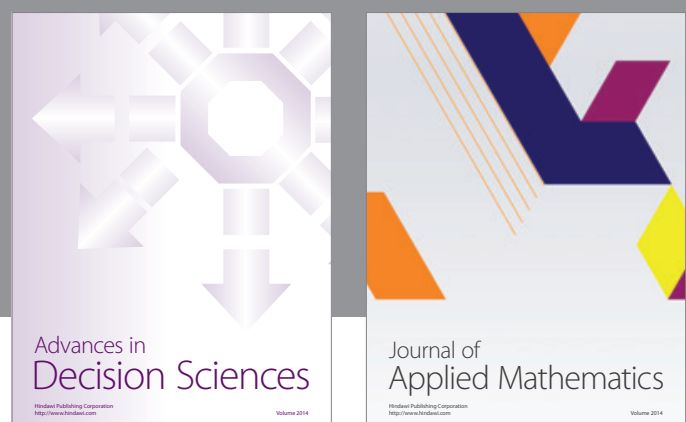

Journal of

Applied Mathematics
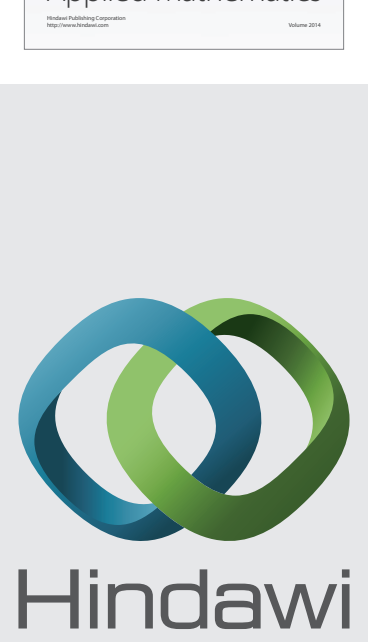

Submit your manuscripts at http://www.hindawi.com
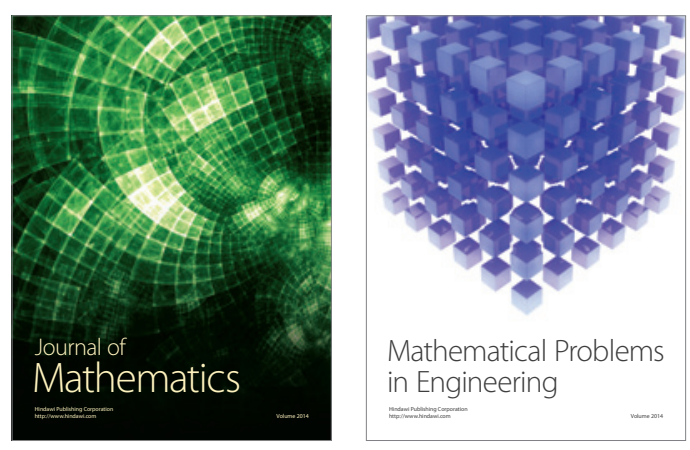

Mathematical Problems in Engineering
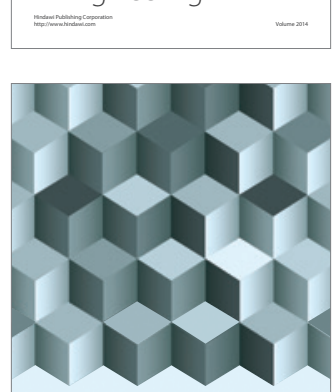

Journal of

Function Spaces
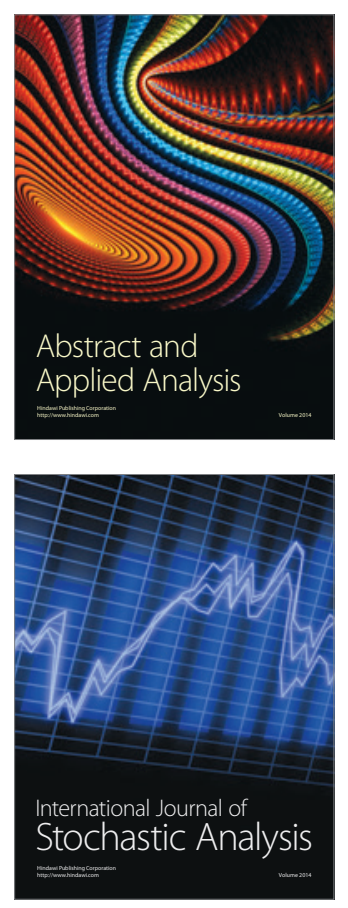

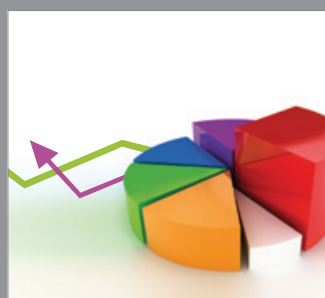

ournal of

Probability and Statistics

Promensencen
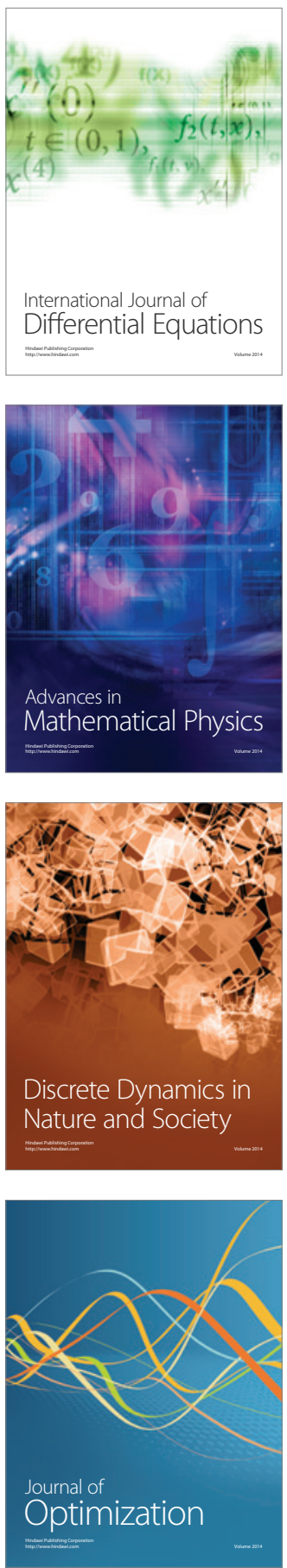